\title{
Studying Strangeness Production with HADES
}

\author{
Heidi Schuldes ${ }^{1, \star}$ for the HADES Collaboration \\ ${ }^{1}$ Goethe-University Frankfurt
}

\begin{abstract}
The High-Acceptance DiElectron Spectrometer (HADES) operates in the $1-2 A \mathrm{GeV}$ energy regime in fixed target experiments to explore baryon-rich strongly interacting matter in heavy-ion collisions at moderate temperatures with rare and penetrating probes. We present results on the production of strange hadrons below their respective $N N$ threshold energy in $\mathrm{Au}+\mathrm{Au}$ collisions at $1.23 \mathrm{~A} \mathrm{GeV}\left(\sqrt{s_{N N}}=2.4 \mathrm{GeV}\right)$. Special emphasis is put on the enhanced feed-down contribution of $\phi$ mesons to the inclusive yield of $K^{-}$and its implication on the measured spectral shape of $K^{-}$. Furthermore, we investigate global properties of the system, confronting the measured hadron yields and transverse mass spectra with a Statistical Hadronization Model (SHM) and a blastwave parameterization, respectively. These supplement the world data of the chemical and kinetic freeze-out temperatures.
\end{abstract}

\section{Introduction}

The HADES mission is to do detailed studies of the properties of strongly interacting matter at high net-baryon densities with rare and penetrating probes. Whereas at low baryo-chemical potential $\mu_{b}$ ab-inito calculations from lattice QCD are predicting a smooth cross-over from deconfined to hadronic matter, calculations at high $\mu_{b}$ are not reliable when based on first principles due to the fermion determinant sign problem [1]. Therefore, hadron properties have to be extracted from models based on effective Lagrangians.

A phenomenological tool to characterize the system produced in Heavy-Ion Collisions (HIC) at various beam energies is the Statistical Hadronization Model (SHM) [2-4]. It allows to extract freeze-out parameters by fitting particle yields. These parameters show a remarkable regularity of falling on a smooth curve in the temperature $T-\mu_{B}$ plane from lowest SIS18 energies up to highest available energies at LHC [5,6]. Whereas at highest energies the appearance of chemical equilibrium in the system can be assumed, it is still under discussion in the SIS18 energy regime, where the collision dynamics show long interpenetration times and a large amount of stopping of the colliding nuclei in the reaction zone.

The production of strange hadrons below their respective nucleon-nucleon threshold energies ${ }^{1}$ is a particular suitable probe for the high-density phase of the HIC. As particles carrying strangeness can not be produced in binary $N N$ collisions, the energy to produce these particles has to be provided by the system. In the framework of transport model calculations the necessary energy is accumulated

\footnotetext{
^e-mail: h.schuldes@gsi.de

${ }^{1}$ The thresholds are for $N N \rightarrow N \Lambda K^{+} / K^{0}: \sqrt{s}_{t h r}=2.55 \mathrm{GeV}$, for $N N \rightarrow N N K^{+} K^{-}: \sqrt{s}_{t h r}=2.86 \mathrm{GeV}$ and for $N N \rightarrow$ $N N \phi: \sqrt{s}_{t h r}=2.89 \mathrm{GeV}$
} 
in multi-step processes via intermediate resonances. As kaons carry an anti-strange quark, their coupling to the baryons of the medium is suppressed. Therefore, they are expected to have a rather long mean free path in nuclear matter of $\lambda \approx 5 \mathrm{fm}$ when applying the low-density approximation to measurements of the $K^{+} N$ cross-section in these models and are supposed to transmit information about the hot and dense stage of the HIC undisturbed [7]. Anti-kaons, on the other hand, show a strong coupling to baryons in these models, leading to a complicated and strongly modified spectral function in the medium. Since the suggestion of the possibility of an $\bar{K}$ condensate in dense nuclear matter from Kaplan and Nelson in the 1980ies [8] and possible implications on astrophysical objects, various attempts to extract information about the strength of the suggested attractive $\bar{K} N$ potential in different approaches have been made (see for example [9-14]).

The first data on sub-threshold $K^{-}$production in HIC obtained by the KaoS collaboration revealed that the $K^{-}$and $K^{+}$multiplicities show, despite their different $N N$ threshold energies, a similar increase with collision centrality, whereas the transverse mass spectra of $K^{-}$are significantly softer compared to the ones of $\mathrm{K}^{+}$(for a review of the data see [15]). These two observations found a possible explanation within transport model calculations when assuming the strangeness exchange reaction $\Lambda \pi \rightarrow N K^{-}$, which was predicted by Ko in the early 1980ies [16] to be the dominant production channel for $K^{-}$below threshold [17]. This reaction couples the production of $K^{-}$to $K^{+}$, thus explaining their similar centrality dependence and the later decoupling of $K^{-}$, resulting in the softer spectrum. However, recent data on strangeness production in smaller collision systems indicate that $\phi$ feed-down decays constitute also a sizeable source for $K^{-}$production below the threshold [18-20], which was not taken into account in transport models.

The measurement of a close to complete set of strange hadrons in the same collision system will help to do a detailed characterization of the produced medium, as well as to constrain different model assumptions concerning effective potentials and production mechanisms. In particular the low part of the transverse momentum spectrum of $K_{S}^{0}$ is sensitive to $K N$ potentials employed in transport models, as it is in contrast to the one of $K^{+}$, not affected by the coulomb potential. The detailed comparison of various observables from $K_{S}^{0}$ and $\Lambda$ to different models is subject of a future publication. In these proceedings we discuss the first measurement of $\phi$ so far below the $N N$ threshold and its implications for sequential $\mathrm{K}^{+} / \mathrm{K}^{-}$freeze-out in section 3 and the general freeze-out properties of the system in section 4.

\section{Experimental setup and data analysis}

The presented data has been recorded with the High Acceptance Di-Electron Spectrometer (HADES), located at the SIS18 accelerator of the GSI Helmholtz center for heavy-ion research in Darmstadt, Germany. The detector is composed of six identical sectors, surrounding the beam axis and covering almost the full azimuthal and polar angles between 18 and $85^{\circ}$. For a detailed description of the spectrometer and its components see [21]. The track and momentum reconstruction are performed with a magnet spectrometer consisting of two Mini Drift Chambers (MDC) in front of and two behind a superconducting magnet. In combination with the time-of-flight determination via a scintillator hodoscope (TOF) and a Resistive Plate Chamber (RPC) at the end of the setup, particles can be identified. In order to reduce the contribution of protons and pions to the signal of the rarely produced kaons, the energy loss signal measured in the drift chambers and the TOF detector can be employed. The background is described using a third order polynomial function and is subtracted in an iterative fitting procedure. Unstable neutral hadrons are identified via their decay into charged particles $\left(\Lambda \rightarrow p \pi^{-}, K_{S}^{0} \rightarrow \pi^{+} \pi^{-}, \phi \rightarrow K^{+} K^{-}\right.$). In order to increase the signal-to-background ratio and to reduce the amount of uncorrelated pairs, cuts on the specific decay topology of the weakly decaying $\Lambda$ and $K_{S}^{0}$ are applied. The remaining background is subtracted using the mixed-event technique. 
In total $2.1 \times 10^{9} \mathrm{Au}+\mathrm{Au}$ events at a kinetic energy of $1.23 \mathrm{~A} \mathrm{GeV}$, corresponding to $\sqrt{s_{N N}}=2.4 \mathrm{GeV}$, are used in the analysis, corresponding to the $40 \%$ most central events, with $\left\langle A_{\text {part }}\right\rangle=191 \pm 11$, as has been estimated with Glauber model calculations $[22,23]$. The analysis is performed in small cells in reduced transverse mass $m_{t}-m_{0}$ and rapidity $y$ and in four $\left(K^{+}, K^{0}\right.$ and $\left.\Lambda\right)$ or two $\left(K^{-}\right.$and $\left.\phi\right)$ centrality bins. The raw signal count rates are corrected in each phase space cell for acceptance and efficiency based on detailed simulations of the detector response. As input for the simulations the particles are generated with a thermal distribution and embedded into UrQMD events [24], which serve as realistic background.

The spectra are parameterized by Boltzmann functions of the following form:

$$
\frac{1}{m_{t}^{2}} \frac{d^{2} N}{d m_{t} d y}=c(y) \cdot \exp -\frac{\left(m_{t}-m_{0}\right)}{T_{B}(y)},
$$

with a rapidity dependent normalization $c(y)$ and the slope parameter $T_{B}(y)$, in order to extrapolate to unmeasured transverse mass regions and to extract the so-called effective temperature of the particles $T_{\text {eff }}=T_{B}\left(y_{c m}\right)$. The resulting rapidity density distributions are extrapolated to rapidity regions not covered by the spectrometer using Gaussian functions to extract the total multiplicities of the particles. For a detailed description of the reconstruction of strange hadrons see [27-29].

\section{$3 \phi$ production and implications for sequential $K^{+} / K^{-}$freeze-out}

The measured $\phi / K^{-}$multiplicity ratio for the $0-40 \%$ most central collisions [27] is shown on the left side of Fig. 1 in comparison with measurements at similar energies in lighter systems [18-20] as well as data at higher $\sqrt{s_{N N}}$ [30-32]. Whereas this ratio is flat at energies above $\sqrt{s_{N N}} \geq 4 \mathrm{GeV}$ with values around 0.15 , it is increasing for energies below the $N N$ threshold. As a consequence $\phi$ feed-down becomes a sizeable source for $K^{-}$production below the threshold in the large Au+Au system.

The estimated multiplicities of all strange hadrons show, despite their different elementary production thresholds, a similar dependence on the centrality of the collision, as observed also at higher energies. Whereas the coupling of $K^{-}$to $K^{+}$, and to $K_{S}^{0}$ and $\Lambda$ due to strangeness conservation, can be explained in a microscopic picture by strangeness exchange reactions, most transport models are not able to reproduce the large $\phi / K^{-}$multiplicity ratio below the $N N$ threshold and can not explain the centrality independence seen for $\phi$ production.

As observed at slightly higher energies [15], we obtained a systematic lower effective temperature for $\mathrm{K}^{-}$than for $\mathrm{K}^{+}$and the other strange hadrons. However, when taking the $25 \%$ feed-down contribution to the $K^{-}$production from $\phi$ decays into account, we find that the $K^{-}$originating from these decays are significantly cooler than thermally produced kaons, and therefore result in an overall softer $K^{-}$spectrum. The effect can be seen on the right side of Fig. 1, where a simulated two-component cocktail of the $K^{-}$transverse mass spectrum around mid-rapidity is shown. To the $75 \%$ thermally produced $K^{-}$(dotted red), generated with the measured effective temperature of the $K^{+}$in the $0-40 \%$ most central collisions [27] $\left(T_{\text {eff }}^{K+}=(104 \pm 1 \pm 1) \mathrm{MeV}\right)$, we add $25 \% K^{-}$from $\phi$ decays (dashed blue), which have been generated according to the measured value of $\phi\left(T_{\text {eff }}^{\phi}=(108 \pm 7) \mathrm{MeV}\right)$. As a consequence the obtained inverse slope parameter of the cocktail (solid black) is significantly lower for $K^{-}$than the one of $K^{+}\left(T_{\mathrm{eff}}^{K-}=84 \mathrm{MeV}\right.$, obtained using Eq. 1), as observed in the experiment (measured $\left.T_{\mathrm{eff}}^{K-}=(84 \pm 6) \mathrm{MeV}\right)$.

In conclusion, we see no indication for a sequential kaon freeze-out scenario, which has been suggested formerly by transport model calculations as an explanation for the significantly lower effective temperature of $K^{-}$compared to $K^{+}$, when simply taking into account the strong $\phi$ feed-down contribution. As a consequence, conclusions about an attractive $K^{-} N$ potential and its implications on production mechanisms and astrophysical objects need to be revisited. 

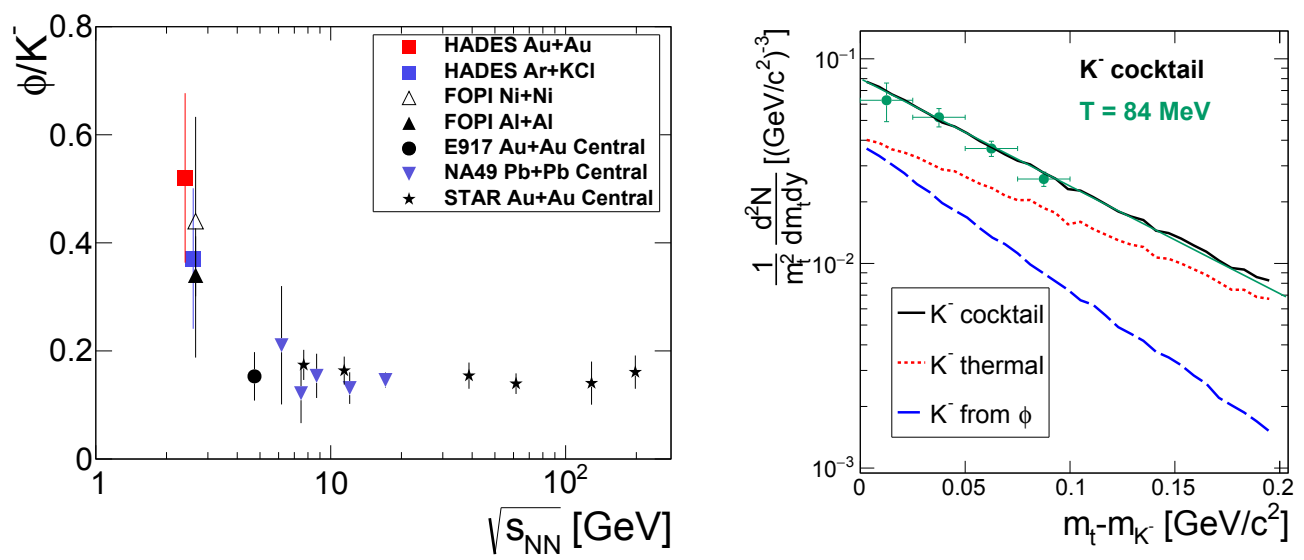

Figure 1. Left: $\phi / K^{-}$multiplicity ratio as a function of $\sqrt{s_{N N}}$ [18-20, 27, 30-32]. Right: Simulated transverse mass spectra of thermally produced $K^{-}$(dotted red), $K^{-}$from $\phi$ decays (dashed blue), and their sum, here denoted as cocktail (black), generated according to the effective temperatures measured for $0-40 \%$ most central collisions. The spectra can be described by Eq. 1 in order to extract the effective temperature $T_{\text {eff }}$.

\section{Freeze-out parameters}

The universal centrality dependence of the yields of strange hadrons indicates that the produced system shows some macroscopic behavior. We find a good description of the measured hadron yields in $20 \%$ most central collisions with a SHM fit based on a mixed-canonical ensemble. The model assumes particle emission from a homogenous source which is in thermal equilibrium, therefore the quantum numbers, except strangeness, are calculated grand canonically. As strangeness is produced very rarely in the analyzed energy regime (typical one $s \bar{s}$ pair per event), its production is additionally suppressed by introducing a sub-volume, characterized by the canonical suppression radius $R_{C}$, in which strangeness has to be conserved exactly and not on average. This suppression is only affecting the open strangeness and not the $\phi$ production, therefore this model is able to describe the enhanced $\phi / K^{-}$ratio. A chemical freeze-out temperature of $T_{\mathrm{ch}}=(68 \pm 2) \mathrm{MeV}$ is extracted.

Furthermore, we find a fair description of the transverse mass spectra at mid-rapidity $\left(y_{c m}^{A u+A u}=0.74\right)$ of protons, kaons and the high energy tail of $\pi^{-}$, for the $10 \%$ most central collisions, by a blast-wave parameterization according to [34]

$$
\frac{d N}{m_{t} d m_{t}} \propto \int_{0}^{R} r d r m_{t} I_{0}\left(\frac{p_{t} \sinh \rho(r)}{T_{\text {kin }}}\right) \times K_{1}\left(\frac{m_{t} \cosh \rho(r)}{T_{\text {kin }}}\right),
$$

where $\rho(r)=\tanh ^{-1} \beta$ and $I_{0}$ and $K_{1}$ are the modified Bessel functions. We employ the radial flow velocity profile of the form

$$
\beta=\beta_{S}(r / R)^{n},
$$

where $\beta_{S}$ is the surface velocity, $r / R$ the relative position in the radial source and $n=1$ the linear flow velocity profile, shown on the right side of Fig. 2 . The averaged radial flow velocity is given by

$$
\langle\beta\rangle=\frac{2}{2+n} \beta_{S} .
$$

We obtain the freeze-out parameters $T_{\text {kin }}=(62 \pm 10) \mathrm{MeV}$ and $\langle\beta\rangle=0.36 \pm 0.04 . \quad \Lambda$ and $\phi$ are slightly deviating from the simultaneous fit, indicating that these particles are preferentially emitted 

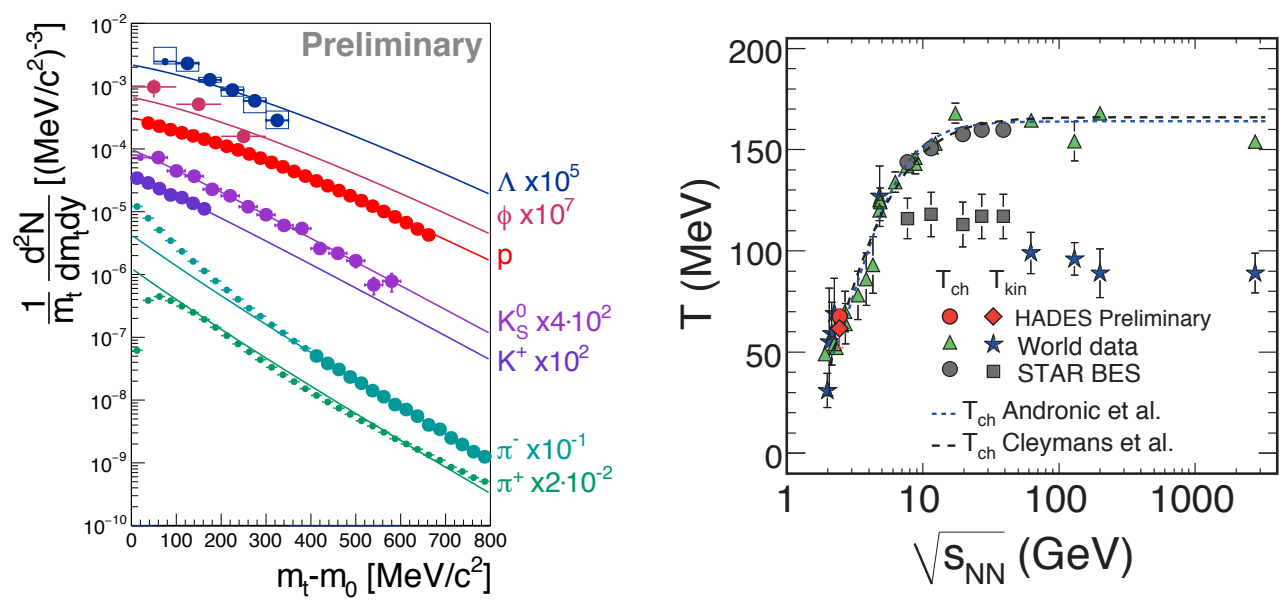

Figure 2. Left: Simultaneous fit to the transverse mass spectra at mid-rapidity of hadrons by a blast-wave parameterization according to Eq. 2. Only fat points are included in the fit. Right: Chemical $\left(T_{\text {ch }}\right)$ and kinetic $\left(T_{\text {kin }}\right)$ freeze-out temperatures obtained in $\mathrm{Au}+\mathrm{Au}$ collisions with HADES (red) compared to world data (summarized in [35]).

from a source with higher temperature and less radial flow.

If we compare the obtained freeze-out temperatures to the world data (summarized in [35]) as shown on the right side of Fig. 2, we find a nice agreement. The measured $T_{\text {kin }}$ is smaller than $T_{\mathrm{ch}}$, which was opposite in smaller collision systems in the analyzed energy regime [36].

\section{Summary and outlook}

The high-quality data on strange hadron production in $\mathrm{Au}+\mathrm{Au}$ collisions at $1.23 \mathrm{~A} \mathrm{GeV}$ measured by HADES allow to characterize the strongly-interacting medium produced in heavy-ion collisions at high net-baryon densities. We find good agreement of the measured hadron yields by a fit with the SHM, assuming a mixed-canonical ensemble in which strangeness is canonically suppressed by $R_{C}$. The extracted chemical freeze-out temperature of $T_{\mathrm{ch}}=(68 \pm 2) \mathrm{MeV}$ is in good agreement with the world data, as well as the kinetic freeze-out temperature $T_{\text {kin }}=(62 \pm 10) \mathrm{MeV}$, extracted from a simultaneous fit to the measured transverse mass spectra of hadrons using a blast-wave approach. Furthermore, we find an enhanced $\phi / K^{-}$multiplicity ratio. Taken this into account, we find a natural explanation of the lower effective temperature measured for $K^{-}$compared to $K^{+}$without assuming a sequential freeze-out scenario of the two kaon species. In conclusion, our results on strangeness production are consistent with the assumption of reaching thermal equilibrium also at SIS18 energies.

In 2018/2019 HADES will continue its broad physics program at the SIS18 accelerator in the context of the FAIR phase-0, focusing on the production of multi-strange hadrons and intermediate-mass dileptons in $\mathrm{Ag}+\mathrm{Ag}$ reactions at $1.65 \mathrm{~A} \mathrm{GeV}$, as well as studying the electromagnetic structure of baryonic resonances in pion-induced reactions. In the later future this research will be continued at higher energies at the SIS100 accelerator. 


\section{References}

[1] O. Philipsen, EPJ Web Conf. 137 (2017) 03016.

[2] P. Braun-Munzinger, K. Redlich, J. Stachel, arXiv:nucl-th/0304013 (2003).

[3] A. N. Tawfik, Int. J. Mod. Phys. A 2917 (2014) 1430021.

[4] M. Floris, Nucl. Phys. A 931 (2014) 103.

[5] J. Cleymans, H. Oeschler, K. Redlich, S. Wheaton, Phys. Rev. C 73 (2006) 034905.

[6] J. Stachel, A. Andronic, P. Braun-Munzinger, K. Redlich, J. Phys. Conf. Ser. 509 (2014) 012019.

[7] C. Hartnack, H. Oeschler, Y. Leifels, E. L. Bratkovskaya, J. Aichelin, Phys. Rept. 510 (2012) 119.

[8] D. B. Kaplan, A. E. Nelson, Phys. Lett. B 175 (1986) 57.

[9] C. H. Lee, G. E. Brown, D. P. Min, M. Rho, Nucl. Phys. A 585 (1995) 401.

[10] J. Schaffner-Bielich, J. Bondorf, A. Mishustin, Nucl. Phys. A 625 (1997) 325.

[11] M. F. M. Lutz, A. Steiner, W. Weise, Nucl. Phys. A 574 (1994) 755.

[12] V. Koch, Phys. Lett. B 337 (1994) 7.

[13] W. Cassing, E. L. Bratkovskaya, U. Mosel, S. Teis, A. Sibirtsev, Nucl. Phys. A 614 (1997) 415.

[14] D. Cabrera, L. Tolos, J. Aichelin, E. Bratkovskaya, J. Phys. Conf. Ser. 668 no.1 (2016) 012048.

[15] A. Förster, F. Uhlig, I. Bottcher, D. Brill, M. Debowski et al. [KaoS Collaboration], Phys. Rev. C 75 (2007) 024906.

[16] C. M. Ko, Phys. Lett. B 120 (1983) 294.

[17] C. Hartnack, H. Oeschler, Y. Leifels, E. L. Bratkovskaya, J. Aichelin, Phys. Rept. 510 (2012) 119.

[18] G. Agakishiev et al. [HADES Collaboration], Phys. Rev. C 80 (2012) 025209.

[19] K. Piasecki et al. [FOPI Collaboration], Phys. Rev. C 91 (2015) 054904.

[20] P. Gasik et al. [FOPI Collaboration], Eur. Phys. J. A 52 (2016) 177.

[21] G. Agakishiev et al. [HADES Collaboration], Eur. Phys. J. A 41 (2009) 243.

[22] R. J. Glauber, G. Matthiae, Nucl. Phys. B 21 (1970) 135.

[23] B. Kardan, Diploma Thesis, Goethe-University Frankfurt (2016).

[24] S.A. Bass et al., Prog. Part. Nucl. Phys.41 (1998) 255-369.

[25] W. Cassing, E.L. Bratkovskaya, Phys. Rep. 308 (1999) 65-233.

[26] C. Hartnack, R.K. Rajeev, J. Aichelin, J. Konopka, S.A. Bass et al., Eur. Phys. J. A 1 (1998) 151-169.

[27] J. Adamczewski-Musch et al. [HADES Collaboration], arXiv:nucl-ex/1703.08418 (2017).

[28] H. Schuldes, PhD Thesis Goethe-University Frankfurt (2016).

[29] T. Scheib, PhD Thesis Goethe-University Frankfurt (2017).

[30] B. Holzman et al. [E917 Collaboration], Nucl. Phys. A 698 (2002) 643.

[31] S. V. Afanasiev et al. [NA49 Collaboration], Phys. Lett. B 491 (2000) 59.

[32] J. Adams et al. [STAR Collaboration], Phys. Lett. B 612 (2005) 181.

[33] S. Wheaton, J. Cleymans, Comput. Phys. Commun. 180 (2009) 84.

[34] E. Schnedermann, J. Sollfrank, U. W. Heinz, Phys. Rev. C 48 (1993) 2462.

[35] L. Adamczyk et al. [STAR Collaboration], arXiv:nucl-ex/1701.07065 (2017).

[36] G. Agakishiev et al. [HADES Collaboration], Eur. Phys. J. A 47 (2011) 21. 